\title{
Optimal subset of signature miRNAs consisting of 7 miRNAs that can serve as a novel diagnostic and prognostic predictor for the progression of cervical cancer
}

\author{
CAN SHI，YIJUN YANG，LEI ZHANG，TING ZHANG，JUANPENG YU, \\ SHANSHAN QIN and YINGCHUN GAO
}

Department of Obstetrics and Gynecology, The Affiliated Huaian No. 1 People's Hospital of Nanjing Medical University, Huaiyin, Huai'an, Jiangsu 223300, P.R. China

Received July 23, 2018; Accepted February 12, 2019

DOI: 10.3892/or.2019.7097

\begin{abstract}
Cervical cancer is the second most commonly diagnosed cancer in women. Novel prognostic biomarkers are required to predict the progression of cervical cancer. Cervical cancer expression data were obtained from The Cancer Genome Atlas (TCGA) database. MicroRNAs (miRNAs) significantly differentially expressed between early- and advanced-stage samples were identified by expression analysis. An optimal subset of signature miRNAs for pathologic stage prediction was delineated using the random forest algorithm and was used for the construction of a cervical cancer-specific support vector machine (SVM) classifier. The roles of signature miRNAs in cervical cancer were analyzed by functional annotation. In total, 44 significantly differentially expressed miRNAs were identified. An optimal subset of 7 signature miRNAs was identified, including hsa-miR-144, hsa-miR-147b, hsa-miR-218-2, hsa-miR-425, hsa-miR-451, hsa-miR-483 and hsa-miR-486. The signature miRNAs were used to construct an SVM classifier and exhibited a good performance in predicting pathologic stages of samples. SVM classification was found to be an independent prognostic factor. Functional enrichment analysis indicated that these signature miRNAs are involved in tumorigenesis. In conclusion, the subset of signature miRNAs could potentially serve as a novel diagnostic and prognostic predictor for cervical cancer.
\end{abstract}

\section{Introduction}

As one of the most frequently diagnosed cancers in women worldwide, it is estimated that cervical cancer accounts

Correspondence to: Dr Yingchun Gao, Department of Obstetrics and Gynecology, The Affiliated Huaian No. 1 People's Hospital of Nanjing Medical University, 1 Huanghe West Road, Huaiyin, Huai'an, Jiangsu 223300, P.R. China

E-mail: gych1342@163.com

Key words: cervical cancer, biomarker, miRNA, prognosis, progression for more than 500,000 new cases and 250,000 deaths each year (1). Human papillomavirus (HPV) infection is recognized as the most significant risk factor that presents in most cervical cancers $(2,3)$. Integration of HPV into the cellular genome causes genome instability, transcriptional variations and epigenetic alterations $(4,5)$. However, HPV alone is not sufficient to induce malignant transformation (6). Therefore, additional cancer-causing genetic variations may underlie the development and progression of cervical cancer.

MicroRNAs (miRNAs) are small, non-coding RNA molecules of $\sim 22$ nucleotides in length, functioning by targeting mRNAs to regulate gene expression at the post-transcriptional level $(7,8)$. miRNAs are involved in diverse biological processes, including the cell cycle, differentiation and metabolism (9). Increasing evidence highlights the involvement of altered miRNA expression in cervical cancer (10). Examples include hsa-miR-21 (11), hsa-miR-196a (12) and hsa-miR-497 (13). hsa-miR-21 is an oncogene overexpressed in cervical cancer, the inhibition of which upregulates the tumor suppressor, programmed cell death 4 (PDCD4) and suppresses cell proliferation (11). Upregulation of hsa-miR-196a has also been detected in cervical cancer tissues, in which it promotes cancer cell proliferation (12). Unlike hsa-miR-21 and hsa-miR-196a, hsa-miR-497 is a tumor suppressor for cervical cancer and suppresses cancer cell migration and invasion by targeting insulin-like growth factor 1 (IGF-1) receptor (13).

Potential prognostic miRNA signatures of cervical cancer have been identified. A miRNA signature for clinical response consisting of hsa-miR-200a and hsa-miR-9 has been previously identified by the expression analysis of candidate miRNAs (14). According to the expression levels of hsa-miR-200a and hsa-miR-9, cervical cancer samples could be divided into low- and high-risk groups. Functional analysis indicated that both hsa-miR-200a and hsa-miR-9 are likely to play important roles in cervical cancer metastasis. However, only a limited number of miRNAs were analyzed in the present study (14), and miRNA expression differences between pathologic stages have not yet been examined.

To identify novel outcome predictors of cervical cancer, we comprehensively analyzed the expression levels of miRNAs using data from The Cancer Genome Atlas (TCGA). miRNAs 
significantly differentially expressed between early (I and II) and advanced (III and IV) pathologic stages were identified, followed by the identification of an optimal subset of signature miRNAs. The subset of signature miRNAs revealed good performance in progression prediction and may serve as a promising prognostic predictor of cervical cancer in clinical practice.

\section{Materials and methods}

Data source. mRNA and miRNA expression profiles (Illumina HiSeq 2000 RNA sequencing data) of cervical cancer were downloaded from TCGA (https://gdc-portal.nci.nih.gov/) database. In addition, the level-3 data in TCGA-CESC was acquired on an online platform (http://gdac.broadinstitute. org/runs/stddata_2016_01_28/data/CESC/20160128/).Samples for which both mRNA and miRNA data as well as information on pathologic stage and survival were selected for the present study. In total, data of 285 samples were collected, which were randomly divided into training (143 samples) and validation (142 samples) datasets. Clinical characteristics of these samples are summarized in Table I.

Screening of significantly differentially expressed miRNAs. Samples in the training dataset were divided into early (I and II) and advanced (III and IV) pathologic-stage groups. miRNA expression profiles were compared between the two groups using the t-test (http://127.0.0.1:26738/library/stats/html/t.test.html)andWilcoxon rank-sum test (http://127.0.0.1:26738/library/stats/html/wilcox. test.html) under R3.1.0. False discovery rate (FDR) $<0.05$ and $\log F C$ (fold change) $>0.263$ were set as thresholds for the selection of significantly differentially expressed miRNAs for both methods. Significantly differentially expressed miRNAs (Table SI) in the two stages were further compared using the t-test in excel. Two-way hierarchical clustering analysis based on centered Pearson correlation (15) was performed using the pheatmap package (https://cran.r-project.org/package=pheatmap) in R. Correlation between clusters and pathologic stages were analyzed by the Chi-square test using the chisq.test function (http://127.0.0.1:21869/library/stats/html/chisq.test.html) in R. Kaplan-Meier survival analysis was conducted for different clusters, using the survival package (version 2.40-1; https://cran.r-project.org/package=survival) under R.

Selection and validation of an optimal subset of a 7-miRNA signature. Signature miRNAs were selected from differentially expressed miRNAs using the bootstrap algorithm (16) of the random forest package (https://cran.r-project.org/ package $=$ randomForest) (17) in R. The optimal subset of a 7-miRNA signature was the one yielding the minimum out-of-bag (OOB) error.

Based on the expression values of the optimal subset of miRNAs, two-way hierarchical clustering analysis was conducted for both the training and the validation dataset, followed by the Kaplan-Meier survival analysis of different clusters.

A cervical cancer-specific support vector machine (SVM) classifier was constructed based on the expression values of the optimal subset of miRNAs, using the SVM function (core function: Sigmoid kernel; cross: 10 -fold cross validation) in the e1701 package in R (18). The SVM classifier was used to predict the pathologic stages of samples. Based on the predictions, samples in both the training and the validation dataset were classified into either an early-stage-like or an advanced-stage-like group. The prognosis of different groups was analyzed using the Kaplan-Meier survival curve analysis.

Independence analysis of an SVM-predicted group as a prognostic factor. Clinical information on age, pathologic $\mathrm{M}$, pathologic $\mathrm{N}$, pathologic $\mathrm{T}$, smoking, new tumor, radiation therapy, and targeted molecular therapy was extracted from both the training and the validation dataset. Correlations between the clinical variables and prognoses were analyzed by univariate and multivariate Cox regressions, using the survival package under R3.1.0. Clinical variables with $\mathrm{P}<0.05$ were considered to be significant and independent prognostic factors.

Samples in both the training and the validation dataset were further stratified according to different clinical variables. The relation between the SVM-predicted group and the prognosis of cervical cancer was analyzed using univariate Cox regression and Kaplan-Meier survival curve analysis for each stratum, with $\mathrm{P}<0.05$ considered to be statistically significant.

Functional analysis of targets of a 7-miRNA signature. mRNAs significantly differentially expressed between early and advanced stages were screened as aforementioned for miRNAs. mRNAs targeted by the optimal subset of a 7-miRNA signature were predicted using miRWalk. The selected miRNA-mRNA pairs could be retrieved in four databases including miRWalk (http://mirwalk.umm.uni-heidelberg.de/), miRanda (19), miRDB (http://www.mirdb.org/), miRMap (https://mirmap. ezlab.org/app/), miRNAMap (http://mirnamap.mbc.nctu. edu.tw/), RNA22 (https://cm.jefferson.edu/rna22/) and TargertScan (http://www.targetscan.org/mamm_31/), which were further intersected with the significantly differentially expressed mRNAs. Thus, the selected mRNAs were used for the construction of a regulatory network of the optimal subset of a 7-miRNA signature. mRNAs in the regulatory network were functionally annotated using DAVID (https://david. ncifcrf.gov/) (20) and significantly enriched Gene Ontology (GO) biological processes, and Kyoto Encyclopedia of Genes and Genomes (KEGG) pathways were retrieved, with $\mathrm{P}<0.05$ set as a threshold for significantly enriched terms.

\section{Results}

Differential miRNA expression between the early- and advanced-stage groups. Among the 143 samples in the training dataset, 90 were early-stage and 53 were advanced-stage samples. miRNAs with low expression (median expression value $<1.0$ ) were removed and the remaining 318 miRNAs were used for further analysis. In total, 51 miRNAs were identified to be significantly differentially expressed between earlyand advanced-stage groups by t-test, whereas 49 miRNAs were identified by Wilcoxon rank-sum test (Fig. 1). In total, 44 miRNAs were identified to be significantly differentially expressed by both t-test and Wilcoxon rank-sum test. 
Table I. Summary of clinical characteristics of the training, validation and entire datasets.

\begin{tabular}{lccc}
\hline Clinical characteristics & Training dataset (N=143) & Validation dataset (N=142) & Entire dataset (N=285) \\
\hline Age (years, mean \pm SD) & $46.65 \pm 13.56$ & $48.92 \pm 13.53$ & $47.78 \pm 13.57$ \\
Pathologic_M (M0/M1/-) & $55 / 4 / 84$ & $50 / 7 / 85$ & $105 / 11 / 169$ \\
Pathologic_N (N0/N1/-) & $62 / 24 / 57$ & $67 / 28 / 47$ & $129 / 52 / 104$ \\
Pathologic_T (T1/T2/T3/T4/-) & $69 / 28 / 9 / 3 / 34$ & $65 / 40 / 9 / 7 / 21$ & $134 / 68 / 18 / 10 / 55$ \\
Pathologic_stage (I/II/III/IV) & $83 / 7 / 43 / 10$ & $72 / 18 / 40 / 12$ & $155 / 25 / 83 / 22$ \\
Smoking (Yes/No/-) & $69 / 56 / 18$ & $56 / 71 / 15$ & $125 / 127 / 33$ \\
New tumors (Yes/No/-) & $24 / 101 / 18$ & $43 / 203 / 39$ \\
Radiation therapy (Yes/No/-) & $88 / 30 / 25$ & $83 / 33 / 26$ & $171 / 63 / 51$ \\
Targeted molecular therapy (Yes/No/-) & $69 / 33 / 41$ & $59 / 30 / 53$ & $128 / 63 / 94$ \\
Status (Deceased/Alive) & $35 / 108$ & $35 / 107$ & $70 / 215$ \\
Overall survival months (mean \pm SD) & $32.07 \pm 39.29$ & $32.25 \pm 38.57$ & $32.16 \pm 38.86$
\end{tabular}

$\mathrm{SD}$, standard deviation.

Table II. Progression-related signature miRNAs of cervical cancer.

\begin{tabular}{|c|c|c|c|c|c|c|}
\hline \multirow[b]{2}{*}{ miRNAs } & \multicolumn{3}{|c|}{ Wilcoxon test } & \multicolumn{3}{|c|}{ t-test } \\
\hline & $\log \mathrm{FC}$ & FDR & P-value & $\log \mathrm{FC}$ & FDR & P-value \\
\hline hsa-miR-144 & 0.701563604 & $4.16 \mathrm{E}-06$ & $1.78 \mathrm{E}-07$ & 0.699709 & $4.46 \mathrm{E}-06$ & $1.95 \mathrm{E}-07$ \\
\hline hsa-miR-147b & -0.654720572 & 0.005331 & 0.000228 & -0.65658 & 0.009557 & 0.000419 \\
\hline hsa-miR-218-2 & -0.40945555 & 0.000328 & $1.40 \mathrm{E}-05$ & -0.41131 & 0.000538 & $2.36 \mathrm{E}-05$ \\
\hline hsa-miR-425 & 0.268232408 & $6.62 \mathrm{E}-06$ & $2.83 \mathrm{E}-07$ & 0.266381 & $1.48 \mathrm{E}-05$ & 6.49E-07 \\
\hline hsa-miR-451 & 0.590364706 & $6.95 \mathrm{E}-07$ & $2.97 \mathrm{E}-08$ & 0.588512 & 7.74E-07 & 3.39E-08 \\
\hline hsa-miR-483 & 0.740086852 & 0.001946 & 8.33E-05 & 0.738239 & 0.00312 & 0.000137 \\
\hline hsa-miR-486 & 0.592724644 & $3.35 \mathrm{E}-06$ & $1.43 \mathrm{E}-07$ & 0.590872 & $8.53 \mathrm{E}-06$ & $3.74 \mathrm{E}-07$ \\
\hline
\end{tabular}

FC, fold change; FDR, false discovery rate.

Two-way hierarchical clustering analysis was carried out based on the expression levels of the 44 miRNAs and separated the training dataset into 2 distinct clusters, designated as clusters 1 and 2 (Fig. 2A). Cluster 1 mainly consisted of early-stage samples, including 86 early- and 15 advanced-stage samples. Cluster 2 mainly consisted of advanced-stage samples, including 38 advanced- and 4 early-stage samples. The overall accuracy of hierarchical clustering in classifying samples was $86.71 \%$ (124 out of 143 samples). Moreover, the 2 clusters were correlated significantly with cancer progression status $\left(\chi^{2}=69.5245, \mathrm{P}<2.2 \mathrm{e}-16\right)$. Kaplan-Meier analysis revealed that samples in cluster 1 were related with a significantly better prognosis than those in cluster 2 (Fig. 2B; log-rank $\mathrm{P}=1.179 \mathrm{e}-05$ ), which was consistent with the dominant sample stages in the respective clusters.

Cervical cancer-related optimal subset of a 7-miRNA signature. The random forest algorithm was used to identify an optimal subset of a 7-miRNA signature from the 44 significantly differentially expressed miRNAs, using the training dataset. The results revealed that the OOB error reached a minimum (0.181) when 7 miRNAs were used for fitting (Fig. 3A). These 7 miRNAs, including miRNAs hsa-miR-144, hsa-miR-147, hsa-miR-218, hsa-miR-425, hsa-miR-451, hsa-miR-483 and hsa-miR-486, were selected as optimal miRNAs and are summarized in Table II. According to the expression levels of these 7 miRNAs in the training dataset, hsa-miR-147 and hsa-miR-218 exhibited a significantly higher expression in the early than in the advanced stage, whereas the remaining 5 miRNAs revealed a significantly lower expression level in the early stage (Fig. 3B).

Hierarchical clustering analysis based on the expression levels of the 7-miRNA signature revealed that samples in the training dataset could be separated into 2 clusters, (Fig. 3C). Similar to the hierarchical clustering results based on the expression levels of all 44 significantly differentially expressed miRNAs, cluster 1 mainly consisted of early-stage samples (74 early- vs. 10 advanced-stage samples) whereas cluster 2 mainly consisted of advanced-stage samples (16 early- vs. 43 advanced-stage samples). The overall 

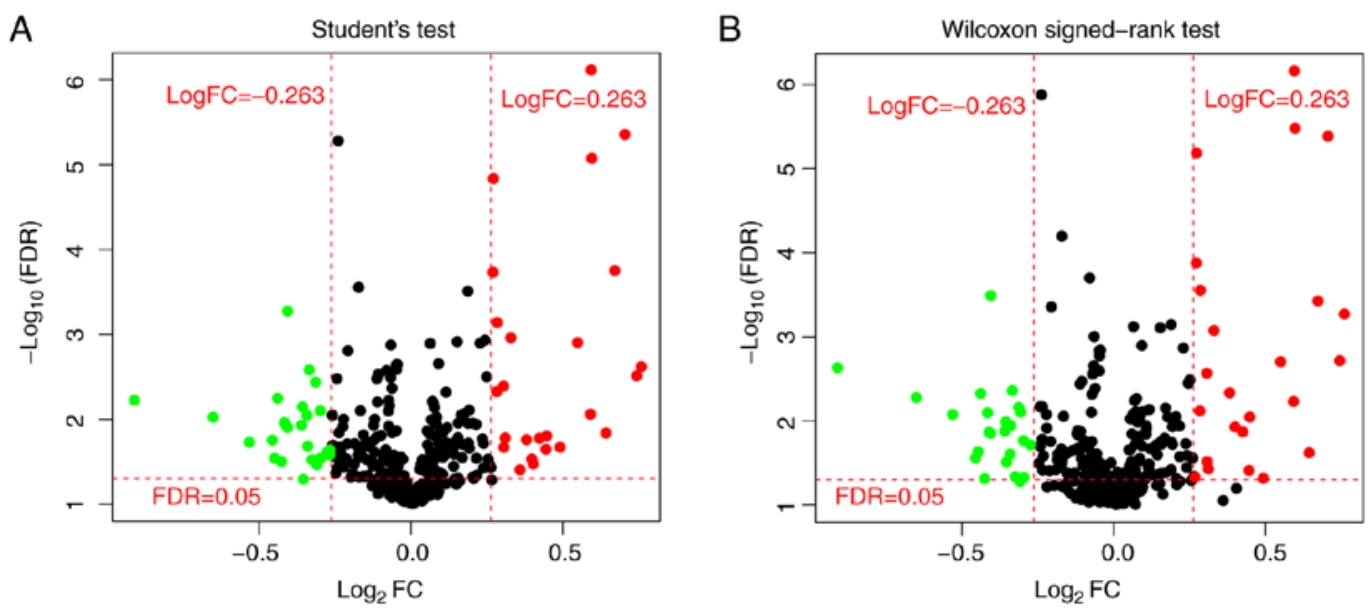

Figure 1. Screening of miRNAs differentially expressed between early- and advanced-stage samples. (A) Volcano plot derived from a t-test. (B) Volcano plot derived from a Wilcoxon rank-sum test. The horizontal red dashed line indicates a FDR $=0.05$ and the vertical red dash line indicates a llogFCl=0.263. The $-\log _{2}(\mathrm{FDR})$ vs. $\log _{2}(\mathrm{FC})$ of each sample was plotted. Red and green dots indicate significantly upregulated and downregulated miRNAs in advanced-stage samples, respectively. FDR, false discovery rate; FC, fold change.
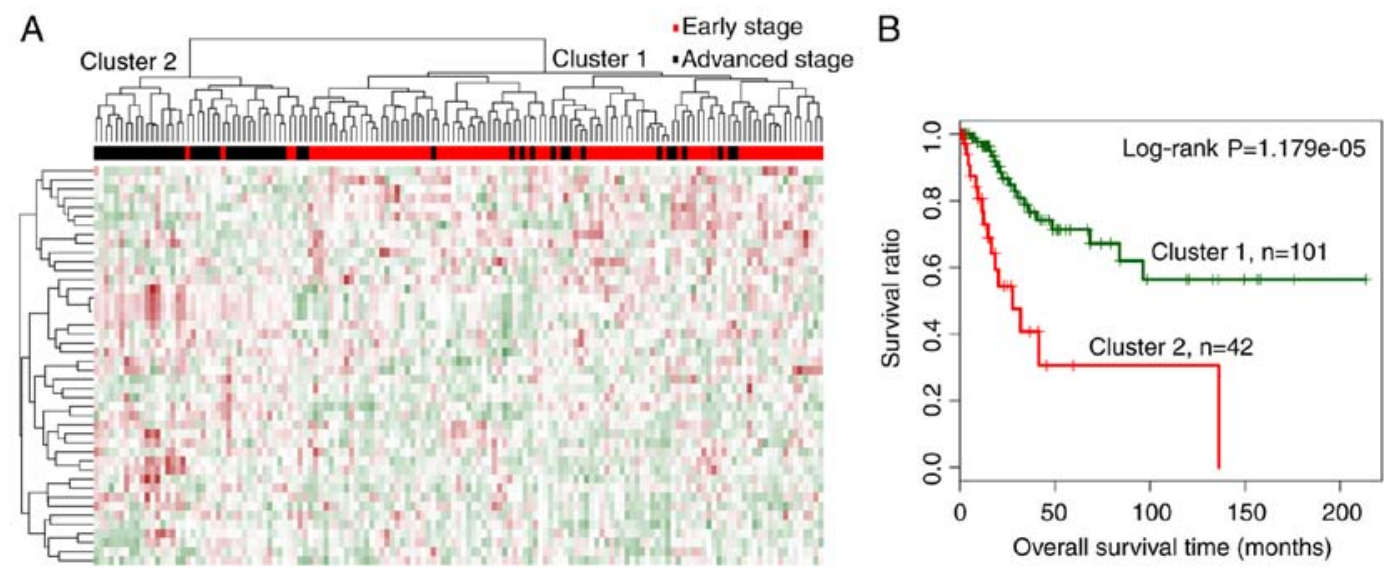

Figure 2. Hierarchical clustering and survival analyses of samples in the training dataset. (A) Two-way hierarchical clustering of samples in the training dataset based on the expression levels of the 44 significantly differentially expressed miRNAs yielded 2 clusters, labeled as cluster 1 and cluster 2 . Early- and advanced-stage samples are indicated in red and black in the horizontal bar. (B) Kaplan-Meier survival curves of the 2 clusters in A. Survival curves for cluster 1 and 2 are indicated in green and red, respectively.

classification accuracy was $81.82 \%$ (117 out of 143 samples). Additionally, cluster 1 was related with significantly better prognosis than cluster 2 (Fig. 3D; log-rank $\mathrm{P}=0.01175$ ).

A cervical cancer-specific SVM classifier for pathologic stage prediction. A cervical cancer-specific SVM classifier was constructed based on the expression levels of a 7-miRNA signature in the optimal subset. Samples in the training dataset were classified as early-stage-like or advanced-stage-like using the SVM classifier. The results revealed that the SVM classifier could classify samples in the training dataset with an overall accuracy of $85.31 \%$ (122 out of 143 samples; sensitivity, $79.81 \%$, specificity, $94.44 \%$, positive prediction value (PPV), $88.095 \%$, negative prediction value (NPV), $84.16 \%$, and area under the receiver operating characteristic (ROC) curve (AUC), 0.897) (Fig. 3E). Kaplan-Meier survival analysis revealed that the predicted early-stage-like group had a significantly better prognosis than the advanced-stage-like group (Fig. 3F; $\log$-rank $\mathrm{P}=0.004715$ ).
Validation of the performance of a 7-miRNA signature in pathologic stage prediction. The validation dataset was used to validate the performance of a 7-miRNA signature in predicting pathologic stage. The validation samples were first classified into cluster 1 and cluster 2 by hierarchical clustering (Fig. 4A). Similar to the results for the training dataset, cluster 1 mainly consisted of early-stage samples (78 early- vs. 15 advanced-st age samples) and cluster 2 mainly consisted of advanced-stage samples (12 early- vs. 37 advanced-stage samples). The overall accuracy was $80.99 \%$ (115 out of 142 samples). Prognosis in the 2 clusters was analyzed using Kaplan-Meier survival curve analysis. Consistent with the findings based on the training dataset (Fig. 4D), classification in cluster 1 indicated significantly better prognosis (Fig. 4B).

Then, we classified the validation samples into earlystage-like and advanced-stage-like groups using the SVM classifier. Consistent with the training dataset findings, the validation samples were classified with an accuracy of $80.98 \%$ (115 out of 142 samples; sensitivity, $73.46 \%$, 
A

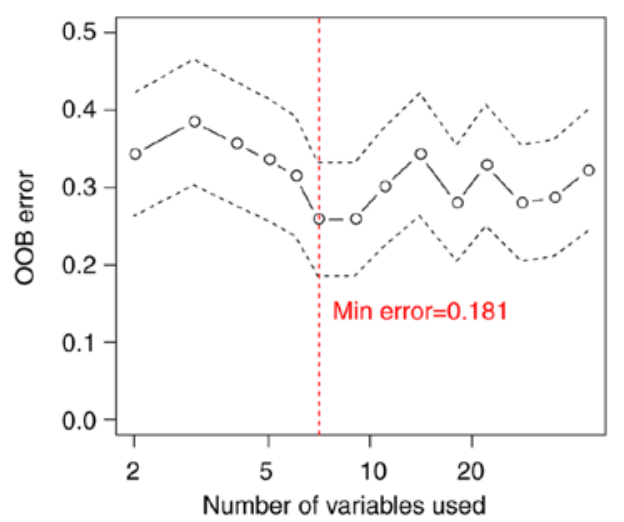

C

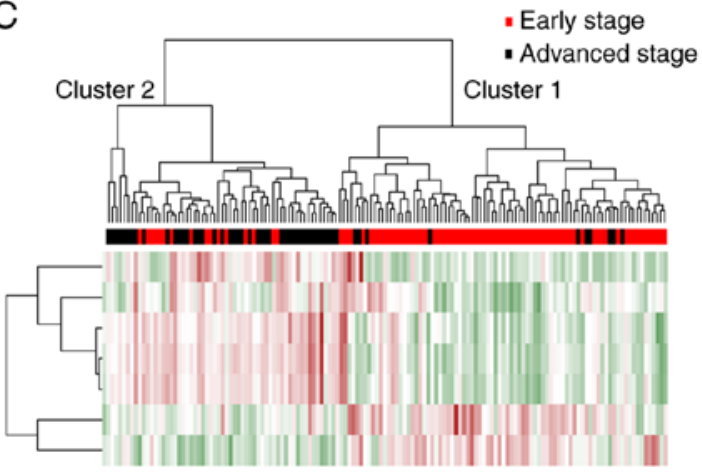

E

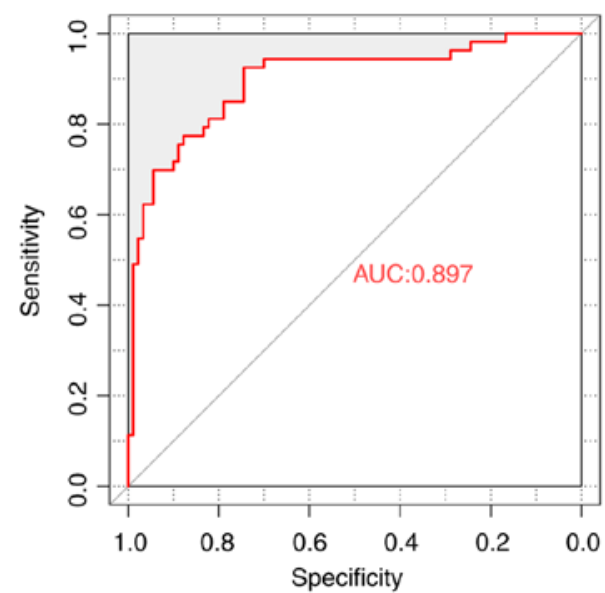

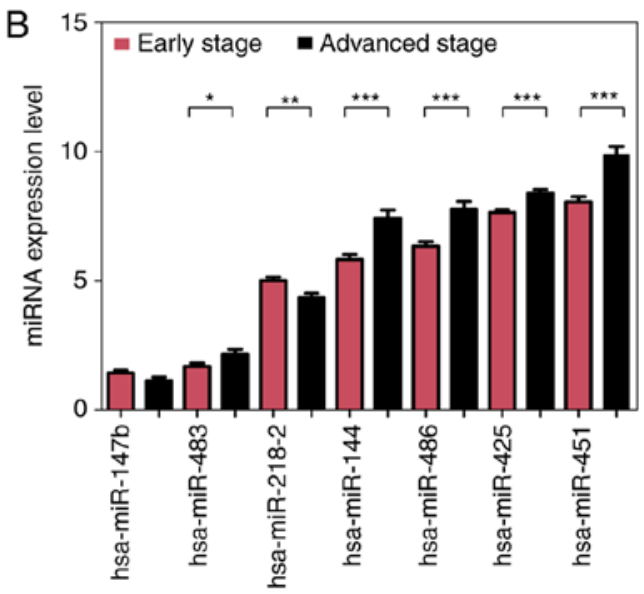
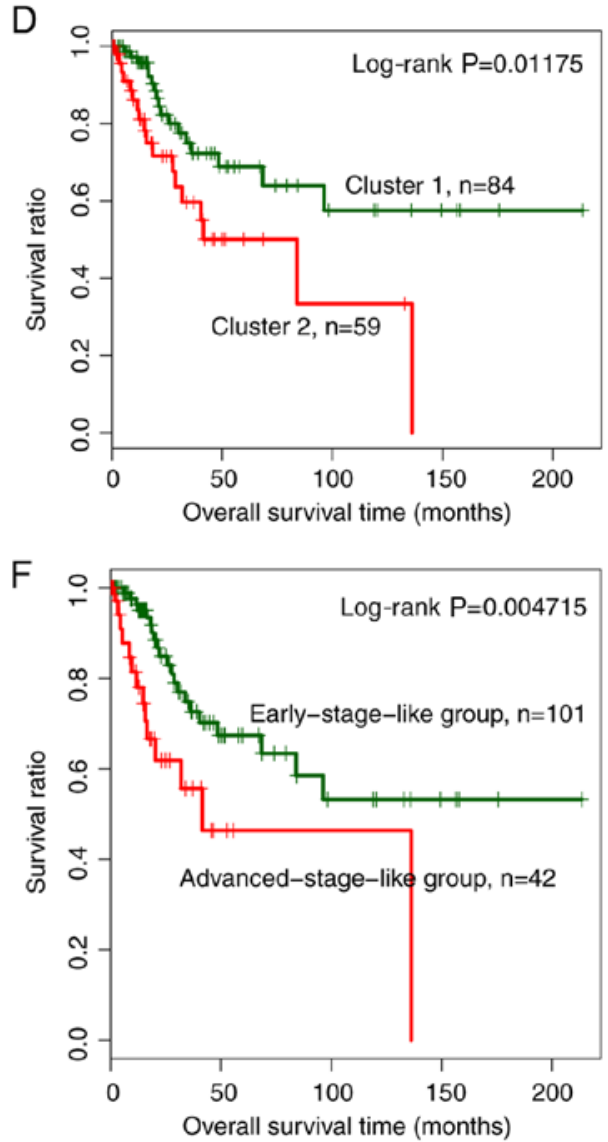

Figure 3. Optimal subset of signature miRNAs and their performance in sample classification. (A) OOB error curve for signature miRNA selection using the training dataset. The OOB error is plotted against the number of signature miRNAs used. The red vertical dashed line indicates the minimum OOB error (0.181), where the number of selected signature miRNAs is 7. (B) Expression levels of the signature miRNAs of the optimal subset in early- (red) and advanced (black)-stage groups using a t-test. ${ }^{*} \mathrm{P}<0.05,{ }^{* *} \mathrm{P}<0.01,{ }^{* * * *} \mathrm{P}<0.005$. (C) Two-way hierarchical clustering of samples in the training dataset based on the expression levels of the 7 signature miRNAs yielded 2 clusters, which are labeled as cluster 1 and cluster 2. Early- and advanced-stage samples are indicated in red and black in the horizontal bar, respectively. (D) Kaplan-Meier survival curves of the 2 clusters in C. Survival curves for clusters 1 and 2 are indicated in green and red, respectively. (E) ROC curves of the training dataset generated using the SVM classifier. The AUC was calculated to be 0.897. (F) Kaplan-Meier survival curves for early-stage-like (green curve) and advanced-stage-like (red curve) groups as predicted by the SVM classifier. OOB, out-of-bag; ROC, receiver operating characteristic; SVM, specific support vector machine; AUC, area under the curve.

specificity, 91.11\%, PPV, 80.49\%, NPV, $81.19 \%$ and AUC, 0.857) (Fig. 4C). Kaplan-Meier survival curve analysis revealed that the early-stage-like samples corresponded with significantly better prognosis than the advanced-stage-like group (Fig. 4D; log-rank $\mathrm{P}=0.006246$ ).

Independence of the SVM-predicted group in progression prediction. Correlations between clinical variables and prognosis were analyzed using Cox regression. Both univariate and multivariate Cox regression revealed that the SVM-predicted group, pathologic T, and new tumors were independent prognostic factors, since they were significantly correlated with prognosis $(\mathrm{P}<0.05)$ in both the training and the validation dataset (Table III). Kaplan-Meier survival curve analysis for both the training and the validation dataset revealed that samples under pathologic $\mathrm{T} 1$ and $\mathrm{T} 2$ categories 
Table III. Cox regression analysis of the correlations between the clinical variables and prognosis for both the training and the validation datasets.

\begin{tabular}{|c|c|c|c|c|c|c|}
\hline \multirow[b]{2}{*}{ Variables } & \multicolumn{3}{|c|}{ Univariate analysis } & \multicolumn{3}{|c|}{ Multivariate analysis } \\
\hline & HR & $95 \% \mathrm{CI}$ & P-value & HR & $95 \%$ CI & P-value \\
\hline \multicolumn{7}{|l|}{ Training dataset $(\mathrm{N}=143)$} \\
\hline $\begin{array}{l}\text { SVM predicted group } \\
\text { (Early/Advanced stage) }\end{array}$ & 2.601 & $1.308-5.172$ & 0.00472 & 2.07 & $0.341-2.587$ & 0.0272 \\
\hline $\begin{array}{l}\text { Age (years) } \\
(\leq 45 />45)\end{array}$ & 1.239 & $0.633-2.426$ & 0.531 & - & - & - \\
\hline $\begin{array}{l}\text { Pathologic M } \\
\text { (M0/M1) }\end{array}$ & - & - & - & - & - & - \\
\hline $\begin{array}{l}\text { Pathologic N } \\
\text { (N0/N1) }\end{array}$ & 4.861 & $1.583-14.93$ & 0.00227 & 3.037 & $0.902-10.22$ & 0.0729 \\
\hline $\begin{array}{l}\text { Pathologic T } \\
(\mathrm{T} 1 / \mathrm{T} 2 / \mathrm{T} 3 / \mathrm{T} 4)\end{array}$ & 2.076 & $1.27-3.395$ & 0.00234 & 2.916 & $1.209-7.032$ & 0.0172 \\
\hline $\begin{array}{l}\text { Smoking } \\
(\text { Yes/No) }\end{array}$ & 1.461 & $0.726-2.944$ & 0.286 & - & - & - \\
\hline $\begin{array}{l}\text { New tumors } \\
(\text { Yes/No) }\end{array}$ & 6.036 & $3.083-11.82$ & 2.60E-09 & 6.101 & $1.964-14.24$ & 0.00053 \\
\hline $\begin{array}{l}\text { Radiation therapy } \\
\text { (Yes/No) }\end{array}$ & 0.9697 & $0.4204-2.237$ & 0.943 & - & - & - \\
\hline $\begin{array}{l}\text { Targeted molecular therapy } \\
\text { (Yes/No) }\end{array}$ & 0.891 & $0.4168-1.904$ & 0.765 & - & - & - \\
\hline \multicolumn{7}{|l|}{ Validation dataset $(\mathrm{N}=142)$} \\
\hline $\begin{array}{l}\text { SVM predicted group } \\
\text { (Early/Advanced stage) }\end{array}$ & 2.473 & $1.265-4.836$ & 0.00625 & 1.233 & $0.422-3.601$ & 0.00702 \\
\hline $\begin{array}{l}\text { Age (years) } \\
(\leq 45 />45)\end{array}$ & 1.248 & $0.628-2.48$ & 0.526 & - & - & - \\
\hline $\begin{array}{l}\text { Pathologic M } \\
\text { (M0/M1) }\end{array}$ & - & - & - & - & - & - \\
\hline $\begin{array}{l}\text { Pathologic N } \\
(\mathrm{N} 0 / \mathrm{N} 1)\end{array}$ & 1.81 & $0.732-4.474$ & 0.193 & - & - & - \\
\hline $\begin{array}{l}\text { Pathologic T } \\
\text { (T1/T2/T3/T4) }\end{array}$ & 1.71 & $1.174-2.491$ & 0.00384 & 1.739 & $1.119-2.701$ & 0.01388 \\
\hline $\begin{array}{l}\text { Smoking } \\
(\text { Yes/No) }\end{array}$ & 1.39 & $0.683-2.827$ & 0.362 & - & - & - \\
\hline $\begin{array}{l}\text { New tumors } \\
(\text { Yes/No) }\end{array}$ & 3.94 & $1.908-8.136$ & $6.32 \mathrm{E}-05$ & 5.229 & $2.117-12.919$ & 0.000337 \\
\hline $\begin{array}{l}\text { Radiation therapy } \\
\text { (Yes/No) }\end{array}$ & 1.155 & $0.517-2.578$ & 0.725 & - & - & - \\
\hline $\begin{array}{l}\text { Targeted molecular therapy } \\
\text { (Yes/No) }\end{array}$ & 1.074 & $0.472-2.446$ & 0.865 & - & - & - \\
\hline
\end{tabular}

HR, hazard ratio; CI, confidence interval.

were related to a significantly better prognosis than those under pathologic T3 and T4 categories, and samples without new tumors were correlated with a better prognosis than those with new tumors (Fig. 5).

Stratified analysis was performed to evaluate the independence of the SVM-predicted group as a prognostic factor.
Samples in both the training and the validation dataset were stratified according to age, pathologic $\mathrm{M}$, pathologic $\mathrm{N}$, pathologic $\mathrm{T}$, smoking, new tumors, radiation therapy and targeted molecular therapy. Univariate Cox regression revealed that the SVM-predicted group was significantly correlated with the prognosis for elder patients ( $>45$ years of age) in both the 

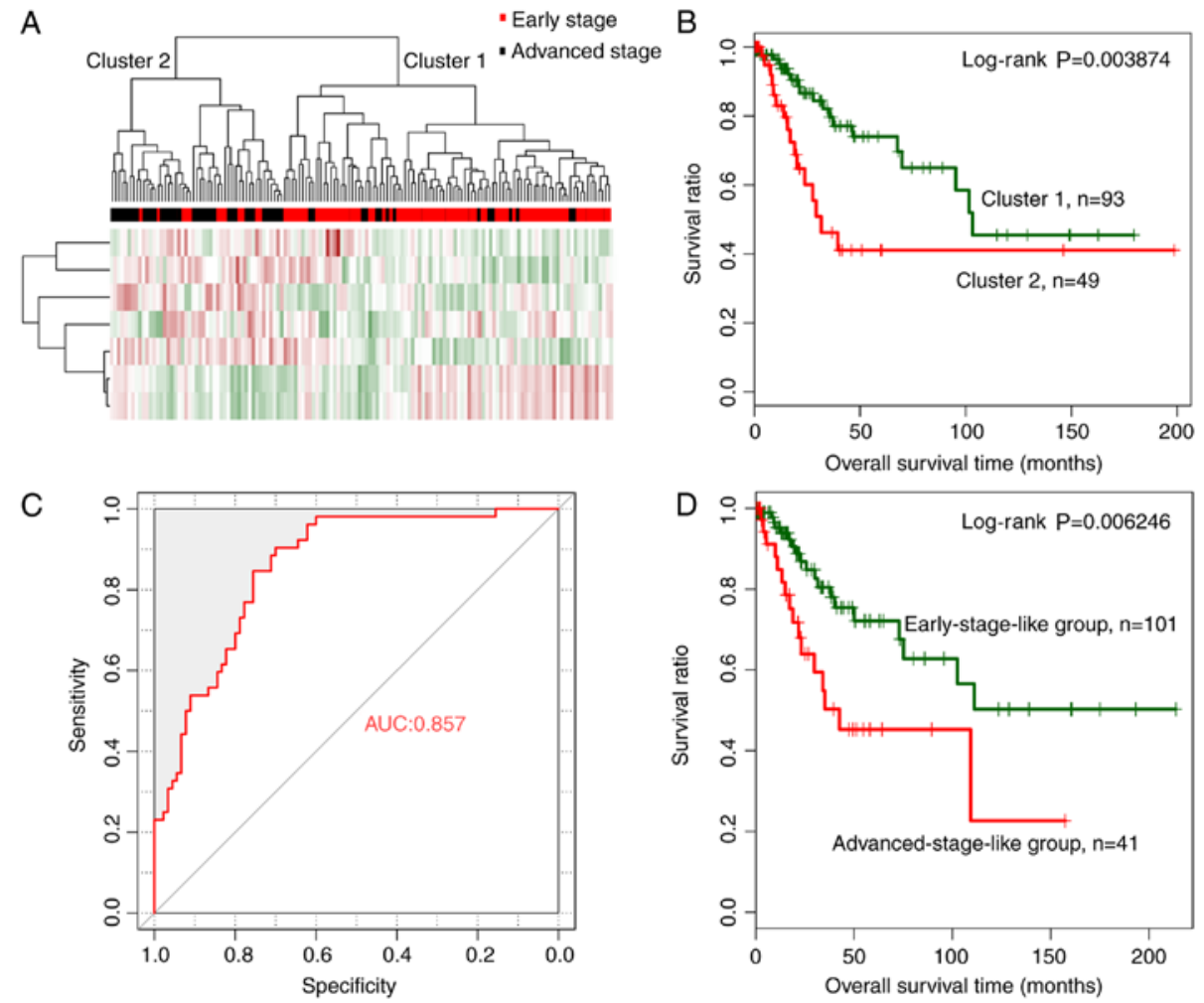

Figure 4. Validation of the optimal subset of signature miRNAs using the validation dataset. (A) Two-way hierarchical clustering of samples in the validation dataset based on the expression levels of the 7 signature miRNAs yielded 2 clusters, which are labeled as cluster 1 and cluster 2. Early- and advanced-stage samples are indicated in red and black in the horizontal bar, respectively. (B) Kaplan-Meier survival curves for clusters 1 (green curve) and 2 (red curve) in A. (C) ROC curves of the validation dataset generated using the SVM classifier. The AUC was calculated to be 0.857. (D) Kaplan-Meier survival curves of early-stage-like (green curve) and advanced-stage-like (red curve) groups classified by the SVM classifier. ROC, receiver operating characteristic; SVM, specific support vector machine; AUC, area under the curve.

A
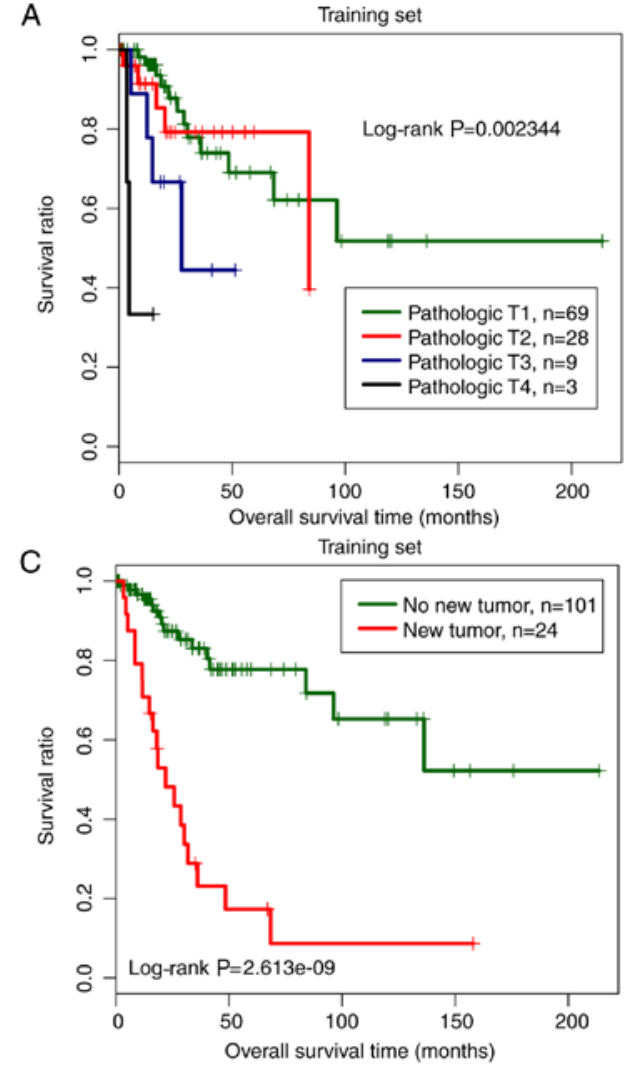

B
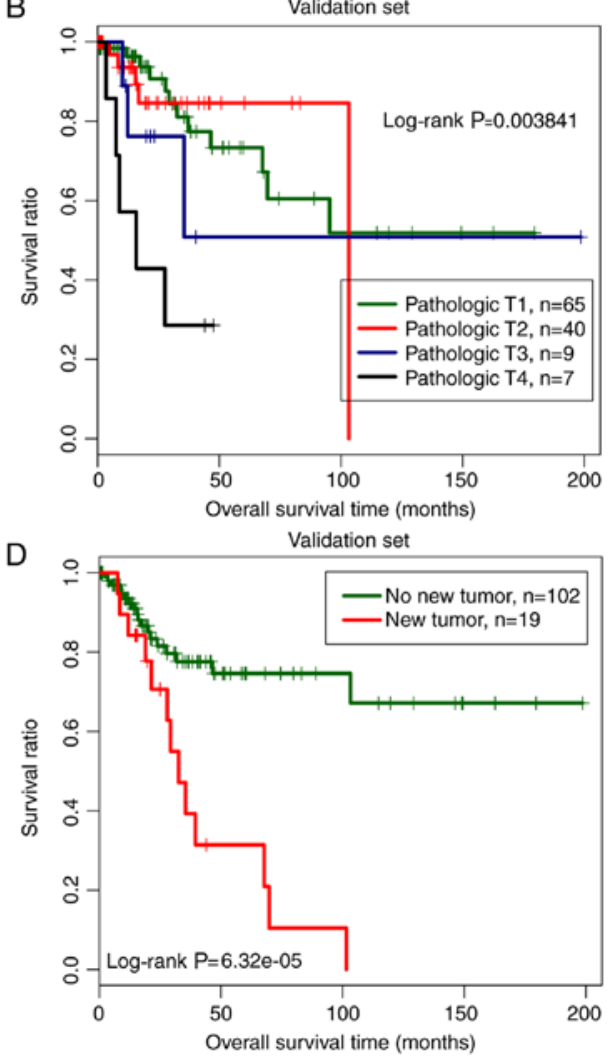

Figure 5. Survival analysis of different pathologic T categories and new tumor status. (A and B) Kaplan-Meier survival curves of different pathologic T categories in the (A) training and the (B) validation datasets. Survival curves for pathologic stages T1, T2, T3 and T4 are shown in green, red, blue and black, respectively. (C and D) Kaplan-Meier survival curves of patients with (red lines) and without (green lines) new tumors in the (C) training and the (D) validation datasets. 
A

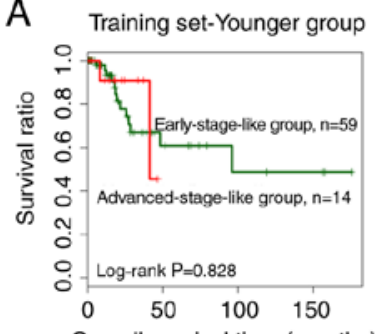

C

C Training set-Pathologic No group

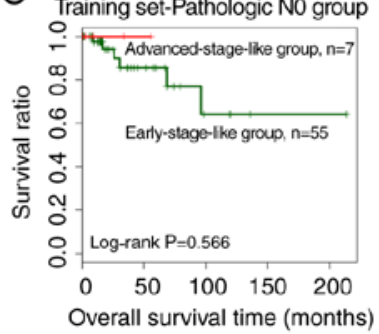

E

E Training set-no new tumor group

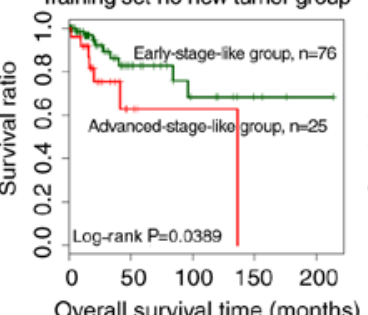

Overall survival time (months)

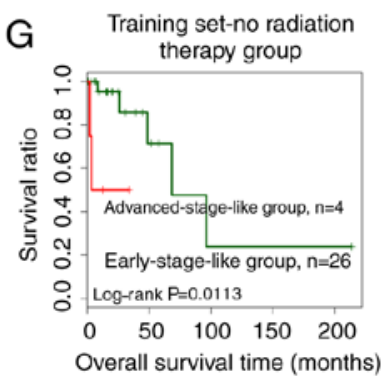

Training set-Elder group
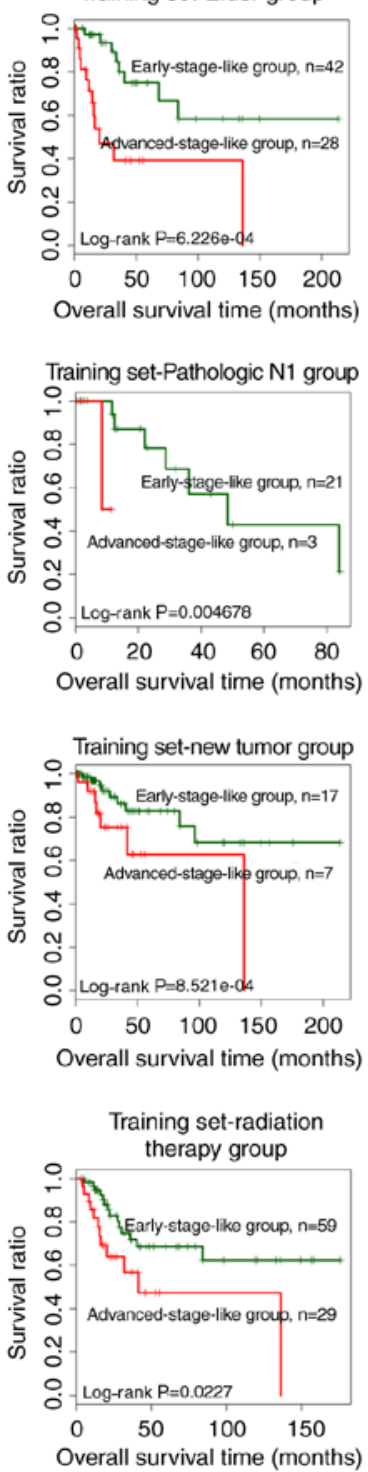

B Validation set-Younger group

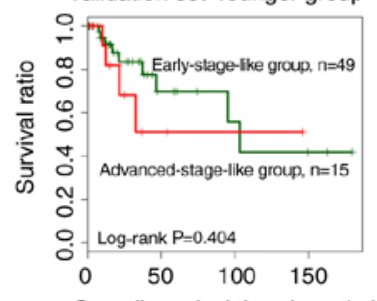

Overall survival time (months)

$D_{\text {Validation set-Pathologic N0 group }}$

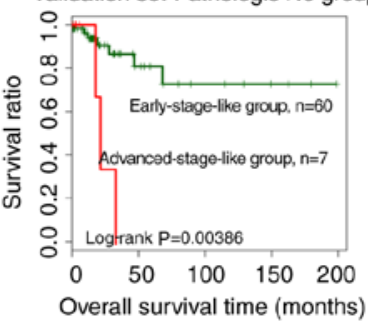

$\mathrm{F}$

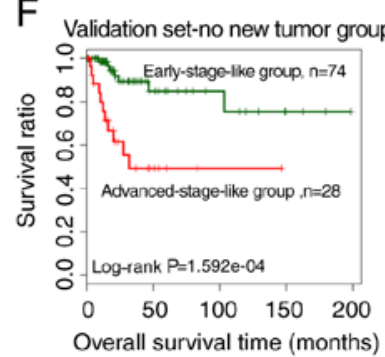

$\mathrm{H} \quad$ Validation set-no radiation

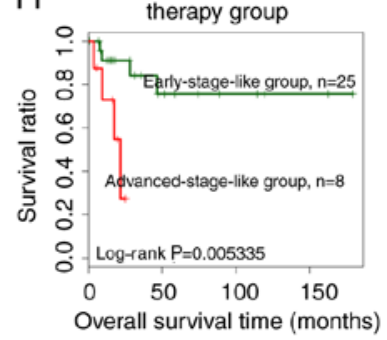

Validation set-Elder group

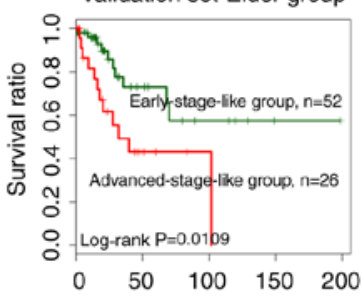

Overall survival time (months)

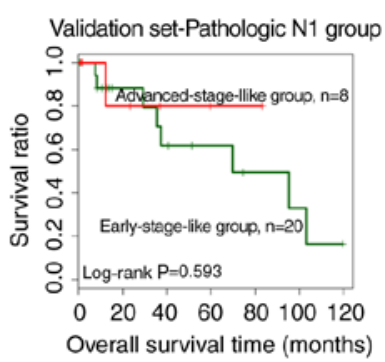

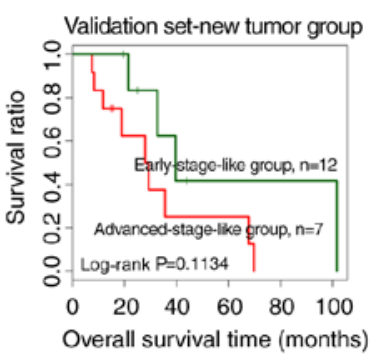

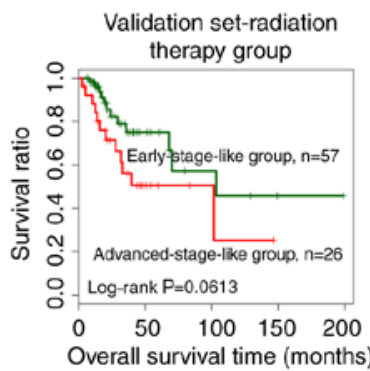

Figure 6. Stratified Kaplan-Meier survival curve analysis. Samples were stratified by (A and B) age, (C and D) pathologic N, (E and F) new tumor, and $(\mathrm{G}$ and $\mathrm{H})$ radiation therapy for both the training and the validation datasets. Survival curves for early-stage-like and advanced-stage-like groups in each stratum are shown in green and red, respectively.

training and the validation dataset (Table IV). Similar results were obtained for patients without new tumors and radiation therapy (Table IV). Additionally, the SVM-predicted group was significantly correlated with pathologic N1 patients in the training dataset and pathologic N0 patients in the validation dataset (Table IV). Kaplan-Meier survival curves for samples stratified by age, pathologic $\mathrm{N}$, new tumors and radiation therapy are presented in Fig. 6.

Functional annotation of a 7-miRNA signature. mRNA expression profiles of early- and advanced-stage samples were compared, and 535 significantly differentially expressed mRNAs were identified. Target mRNAs of a 7-miRNA signature were predicted using miRwalk. As a result, we found that 56, 47, 34, 14, 30 and 31 significantly differentially expressed mRNAs were targeted by hsa-miR-218, hsa-miR-144, hsa-miR-425, hsa-miR-483, hsa-miR-486 and hsa-miR-451, respectively, while none were targeted by hsa-miR-147. We constructed a cervical cancer-related miRNA-mRNA regulation network, consisting of a 7-miRNA signature and significantly differentially expressed mRNAs targeted by this 7-miRNA signature (Fig. 7A). The network consisted of 207 nodes, containing a 6-miRNA signature and 201 significantly differentially expressed target mRNAs.

To interpret the potential roles of the miRNA signature in cervical cancer, functional enrichment analysis was conducted using DAVID (20). The results revealed that mRNAs in the network were significantly enriched for cancer-related GO biological processes and KEGG pathways (Fig. 7B and C). The GO terms included cell-cell signaling, epithelium development, ion transport and adhesion (Fig. 7B). The KEGG terms included pathways in cancer, calcium signaling pathway, basal cell carcinoma, Hedgehog signaling pathway, Wnt signaling pathway, heparan sulfate biosynthesis and TGF- $\beta$ signaling pathway (Fig. 7C). 
Table IV. Stratified prognosis analysis of patients in both the training and the validation datasets.

\begin{tabular}{|c|c|c|c|c|c|c|c|}
\hline \multirow[b]{2}{*}{ Variables } & \multicolumn{3}{|c|}{ Training dataset } & \multirow[b]{2}{*}{ Variables } & \multicolumn{3}{|c|}{ Validation dataset } \\
\hline & HR & $95 \% \mathrm{CI}$ & P-value & & HR & $95 \%$ CI & P-value \\
\hline Age (years) & & & & Age (years) & & & \\
\hline$\leq 45(\mathrm{~N}=73)$ & 0.846 & $0.187-3.825$ & 0.828 & $\leq 45(\mathrm{~N}=64)$ & 1.647 & $0.504-5.388$ & 0.404 \\
\hline$>45(\mathrm{~N}=70)$ & 4.379 & $1.751-10.95$ & 0.000623 & $>45(\mathrm{~N}=78)$ & 2.865 & $1.229-6.684$ & 0.0109 \\
\hline Pathologic N & & & & Pathologic N & & & \\
\hline N0 (N=62) & 1.34 & $0.813-2.506$ & 0.566 & N0 (N=67) & 8.396 & $1.983-35.55$ & 0.00386 \\
\hline $\mathrm{N} 1(\mathrm{~N}=24)$ & 1.321 & $0.571-2.285$ & 0.004678 & N1 (N=28) & 0.565 & $0.0677-4.715$ & 0.593 \\
\hline Pathologic T & & & & Pathologic T & & & \\
\hline $\mathrm{T} 1+\mathrm{T} 2(\mathrm{~N}=97)$ & 3.153 & $0.976-10.18$ & 0.04289 & $\mathrm{~T} 1+\mathrm{T} 2(\mathrm{~N}=105)$ & 2.135 & $0.673-6.769$ & 0.187 \\
\hline $\mathrm{T} 3+\mathrm{T} 4(\mathrm{~N}=12)$ & 1.062 & $0.178-6.326$ & 0.948 & $\mathrm{~T} 3+\mathrm{T} 4(\mathrm{~N}=16)$ & 1.359 & $0.320-5.77$ & 0.676 \\
\hline Smoking & & & & Smoking & & & \\
\hline Yes $(\mathrm{N}=56)$ & 1.697 & $0.635-4.537$ & 0.286 & Yes $(\mathrm{N}=56)$ & 1.873 & $0.719-4.871$ & 0.191 \\
\hline No $(\mathrm{N}=69)$ & 6.558 & $2.119-20.3$ & 0.000186 & No $(\mathrm{N}=71)$ & 2.138 & $0.741-6.174$ & 0.1503 \\
\hline New tumors & & & & New tumor & & & \\
\hline Yes $(\mathrm{N}=24)$ & 4.868 & $1.759-13.47$ & 0.000852 & Yes $(\mathrm{N}=19)$ & 0.3594 & $0.0961-1.345$ & 0.1134 \\
\hline No $(N=101)$ & 2.769 & $1.012-7.576$ & 0.0389 & No $(N=102)$ & 5.213 & 2.006-13.55 & 0.000159 \\
\hline Radiation therapy & & & & Radiation therapy & & & \\
\hline Yes $(\mathrm{N}=88)$ & 2.407 & $1.104-5.246$ & 0.0227 & Yes $(\mathrm{N}=83)$ & 2.12 & $0.948-4.74$ & 0.0613 \\
\hline No $(\mathrm{N}=30)$ & 1.843 & $1.173-6.056$ & 0.0113 & No $(\mathrm{N}=33)$ & 1.884 & $1.422-4.372$ & 0.005335 \\
\hline $\begin{array}{l}\text { Targeted molecular } \\
\text { therapy }\end{array}$ & & & & $\begin{array}{l}\text { Targeted molecular } \\
\text { therapy }\end{array}$ & & & \\
\hline Yes $(\mathrm{N}=69)$ & 2.405 & $0.995-5.812$ & 0.0512 & Yes $(\mathrm{N}=59)$ & 1.643 & $1.389-2.292$ & 0.006816 \\
\hline No $(\mathrm{N}=33)$ & 3.257 & $0.622-7.05$ & 0.139 & No $(\mathrm{N}=30)$ & 2.33 & $0.829-6.541$ & 0.0988 \\
\hline
\end{tabular}

HR, hazard ratio; CI, confidence interval.

\section{Discussion}

Although overwhelming evidence has demonstrated that numerous microRNAs (miRNAs) are probably correlated with cervical cancer (10-14), few studies focused on whether these miRNAs were responsible for the progression of cervical cancer. In the present study, we comprehensively analyzed cervical cancer-related miRNA expression profiles from TCGA database and identified 44 miRNAs significantly differentially expressed between early- and advanced-stage samples. Subsequently, an optimal subset of a 7-miRNA signature was extracted, including hsa-miR-144, hsa-miR-147, hsa-miR-218, hsa-miR-425, hsa-miR-451, hsa-miR-483 and hsa-miR-486. Moreover, both hierarchical clustering and support vector machine (SVM) prediction results demonstrated that a 7-miRNA signature played essential roles in the prediction of cervical cancer progression. Subsequently, the high performance of this subset of 7 miRNAs was validated in hierarchical clustering and SVM prediction using a validation dataset. Additionally, Kaplan-Meier survival analysis revealed that the SVM-predicted early-stage-like group exhibited significant better prognosis than the advanced-stage-like group for both the training and the validation dataset. We also examined the independence of SVM prediction as a prognostic factor. Both univariate and multivariate Cox regression analyses confirmed the independence of the SVM-predicted group in outcome prediction for the training and validation datasets. The prognostic power of SVM prediction was additionally evaluated using stratified analysis. Elder patients ( $>45$ years of age) in both datasets could be classified into early-stage-like and advanced-stage-like groups with significant differences in prognosis. Similar results were acquired for patients with no new tumors and patients that had received no radiation therapy in both datasets. Collectively, the results demonstrated that SVM prediction was a prognostic factor independent of other clinical factors, including age, new tumors and radiation therapy.

miRNAs act by regulating the expression of target mRNAs $(8,9)$. To interpret the functions of the 7-miRNA signature in cervical cancer, potential target mRNAs were identified and subjected to GO and KEGG functional enrichment analyses. Cancer-related GO biological processes and KEGG pathways were overrepresented among these mRNAs, which supported the importance of the 7-miRNA signature in cervical cancer. Two KEGG terms, Hedgehog signaling pathway and Wnt signaling pathway, deserve special attention. Both pathways are essential for cancer cell proliferation, migration, and invasion in various types of cancers, and thus, represent promising targets for cancer therapy $(21,22)$. 

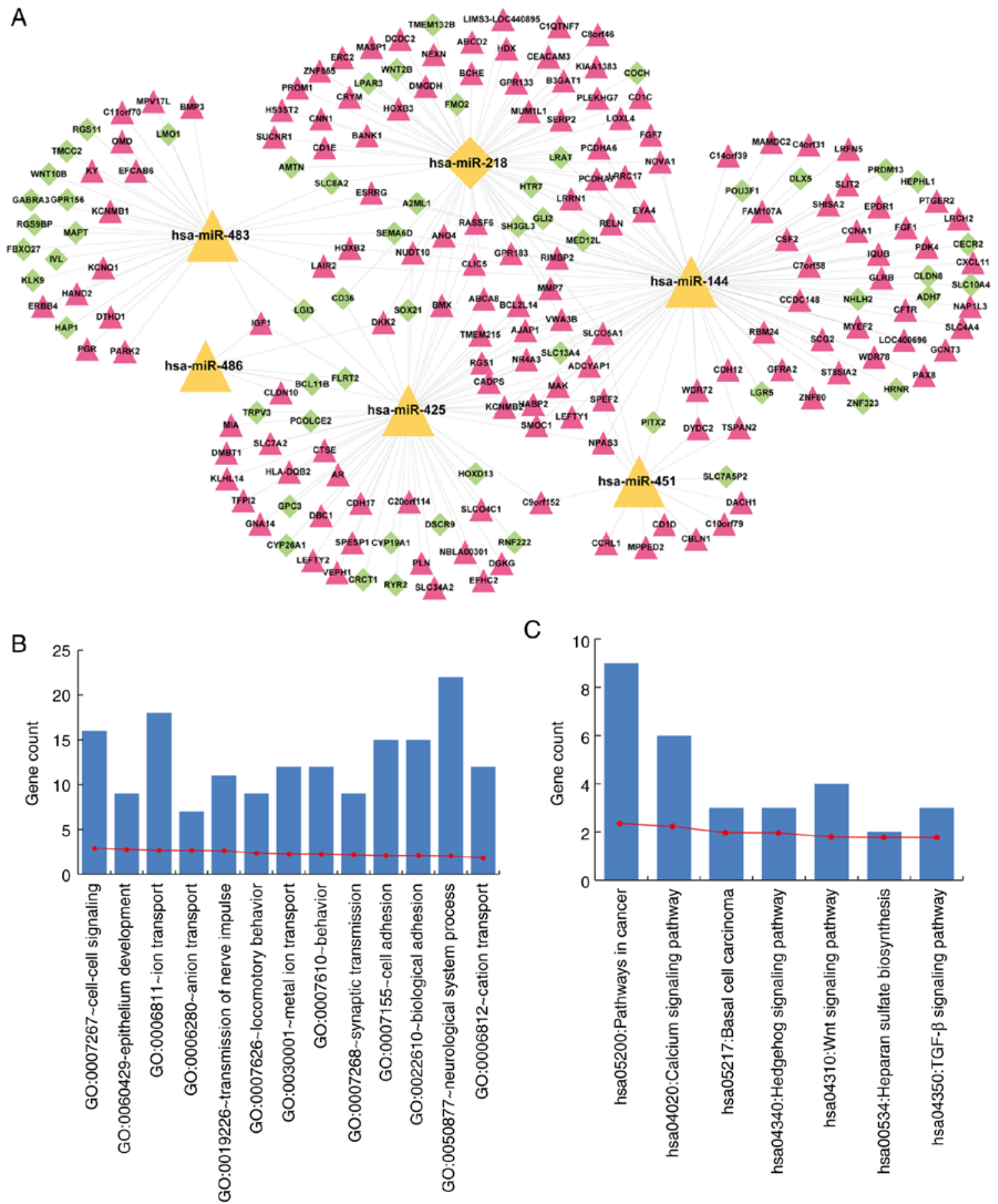

Figure 7. Functional enrichment analysis of mRNAs targeted by the optimal subset of signature miRNAs. (A) Regulatory network consisting of signature miRNAs and their targeting mRNAs. Upregulated and downregulated signature miRNAs in advanced-stage samples are displayed as orange triangles and diamonds, respectively. Upregulated and downregulated mRNAs are shown as pink triangles and green diamonds, respectively. Potential regulatory relationships between signature miRNAs and targeting mRNAs are indicated as gray lines. (B) GO and (C) KEGG term enrichment analysis of mRNAs in A. Gene numbers (vertical axis) are plotted against GO and KEGG terms (horizontal axis). - $\log$ (P-values) are indicated as red dots and lines. GO, Gene Ontology; KEGG, Kyoto Encyclopedia of Genes and Genomes.

Accumulating evidence has suggested that the 7-miRNA signature may be associated with the initiation of cervical cancer and other cancers. hsa-miR-218, as a tumor suppressor, is encoded by slit guidance ligand 2 (SLIT2) (23). It has been reported that the expression of hsa-miR-218 was markedly decreased in the sera from cervical patients compared to the age-matched normal healthy women, and its decreased expression was associated with later stages, cervical adenocarcinoma and lymphatic node metastasis (24). In vitro, miR-218 overexpression inhibited cervical cancer progression by regulating clonogenicity, migration, invasion and metastasis by targeting surviving (25). Consistent with the tumor-suppressive role of hsa-miR-218, we revealed that hsa-miR-218 expression was reduced in advanced-stage patients. With regards to the aberrant expression of miR-218, previous studies indicated that the presence of human papillomavirus (HPV)-16 and HPV-1 
increased E6 protein expression, which play an important role in the rapid ubiquitin-dependent degradation of p53, resulting in reduced expression of miR-218 by suppressing the transcription of SLIT2 $(26,27)$. In addition, hsa-miR-486, hsa-miR-425 and hsa-miR-144 have also been reported to be involved in cervical cancer (28-30). It has been reported that hsa-miR-486-3p acted as a tumor suppressor by inhibiting cell growth and metastasis by targeting ECM1 (28). Sun et al revealed that hsa-miR-425 was significantly upregulated in cervical cancer compared with benign cervical disease patients and healthy controls, and may serve as a prognostic indicator related to high TNM stage and positive lymph node metastasis (29). hsa-miR-144 was significantly downregulated in metastatic cervical cancer (30), although its role remains elusive. The remaining 3 miRNAs have not been reported to be associated with cervical cancer. However, multiple studies have suggested that they play crucial roles in other types of cancer. Dysregulation of hsa-miR-483 has been found in various types of cancer, including adrenocortical (31), prostate cancer (32) and lung adenocarcinoma (33). hsa-miR-451 has been reported to be a tumor suppressor in different cancers $(34,35)$ and a protective effect of hsa-miR-147 has been found in ovarian cancer (36). Collectively, we inferred that the 7-miRNA signature may play important roles in the development and progression of cervical cancer and may serve as a potential biomarker of this disease.

However, there were still multiple limitations in the present study. Firstly, a sophisticated bioinformatics analysis depending on a larger sample size would be required to examine the potential regulatory role of the 7-miRNA signature in cervical cancer. In addition, the corresponding experiments need to be conducted to confirm our predictable results such as several candidate RNA transcripts and critical signaling pathways. Additionally, extensive clinical information is also required to integrate into a comprehensive analysis to decipher the regulatory mechanisms of cervical cancer.

In conclusion, we identified a 7-miRNA signature of cervical cancer by a comprehensive miRNA expression analysis. The 7-miRNA signature was significantly associated with the progression of cervical cancer and was used for the construction of a cervical cancer-specific SVM classifier. The SVM is a promising predictor of progression and outcome. Meanwhile, the 7-miRNA signature may be a novel therapeutic target in future clinical practice. However, further experimental and functional studies are required to reveal the specific roles of these signature miRNAs in cervical cancer.

\section{Acknowledgements}

Not applicable.

\section{Funding}

No funding was received.

\section{Availability of data and materials}

All data generated or analyzed during this study are included in this published article.

\section{Authors' contributions}

CS and YY conceived, designed the research and drafted the manuscript; $\mathrm{LZ}$ and $\mathrm{TZ}$ were responsible for the acquisition of data; LZ and JY analyzed and interpreted the data; TZ, SQ and YG performed the statistical analysis; JY, SQ and YG revised the manuscript for important intellectual content. All authors read and approved the manuscript and agree to be accountable for all aspects of the research in ensuring that the accuracy or integrity of any part of the work are appropriately investigated and resolved.

\section{Ethics approval and consent to participate}

Not applicable.

\section{Patient consent for publication}

Not applicable.

\section{Competing interests}

The authors declare that they have no competing interests.

\section{References}

1. Ferlay J, Soerjomataram I, Dikshit R, Eser S, Mathers C, Rebelo M, Parkin DM, Forman D and Bray F: Cancer incidence and mortality worldwide: Sources, methods and major patterns in GLOBOCAN 2012. Int J Cancer 136: E359-E386, 2015.

2. Walboomers JM, Jacobs MV, Manos MM, Bosch FX, Kummer JA, Shah KV, Snijders PJ, Peto J, Meijer CJ and Muñoz N: Human papillomavirus is a necessary cause of invasive cervical cancer worldwide. J Pathol 189: 12-19, 1999.

3. Doorbar J: Molecular biology of human papillomavirus infection and cervical cancer. Clin Sci 110: 525-541, 2006.

4. Jiménez-Wences H, Peralta-Zaragoza O and Fernández-Tilapa G: Human papilloma virus, DNA methylation and microRNA expression in cervical cancer (Review). Oncol Rep 31: 2467-2476, 2014.

5. Akagi K, Li J, Broutian TR, Padilla-Nash H, Xiao W, Jiang B, Rocco JW, Teknos TN, Kumar B, Wangsa D, et al: Genome-wide analysis of HPV integration in human cancers reveals recurrent, focal genomic instability. Genome Res 24: 185-199, 2014.

6. Schiffman M, Wentzensen N, Wacholder S, Kinney W, Gage JC and Castle PE: Human papillomavirus testing in the prevention of cervical cancer. J Natl Cancer Inst 103: 368-383, 2011.

7. Liz $\mathbf{J}$ and Esteller M: lncRNAs and microRNAs with a role in cancer development. Biochim Biophys Acta 1859: 169-176, 2016.

8. Morris KV and Mattick JS: The rise of regulatory RNA. Nat ReV Genet 15: 423-437, 2014.

9. Garzon R, Calin GA and Croce CM: MicroRNAs in cancer. Annu Rev Med 60: 167-179, 2009.

10. Reshmi G and Pillai MR: Beyond HPV: Oncomirs as new players in cervical cancer. FEBS Lett 582: 4113-4116, 2008.

11. Yao Q, Xu H, Zhang QQ, Zhou H and Qu LH: MicroRNA-21 promotes cell proliferation and down-regulates the expression of programmed cell death 4 (PDCD4) in HeLa cervical carcinoma cells. Biochem Biophys Res Commun 388: 539-542, 2009.

12. Hou T, Ou J, Zhao X, Huang X, Huang Y and Zhang Y: MicroRNA-196a promotes cervical cancer proliferation through the regulation of FOXO1 and p27 ${ }^{\mathrm{Kip} 1}$. Br J Cancer 110: 1260-1268, 2014.

13. Luo M, Shen D, Zhou X, Chen X and Wang W: MicroRNA-497 is a potential prognostic marker in human cervical cancer and functions as a tumor suppressor by targeting the insulin-like growth factor 1 receptor. Surgery 153: 836-847, 2013.

14. Hu X, Schwarz JK, Lewis JS Jr, Huettner PC, Rader JS, Deasy JO, Grigsby PW and Wang X: A microRNA expression signature for cervical cancer prognosis. Cancer Res 70: 1441-1448, 2010. 
15. Li J, Xue W, Lv J, Han P, Liu Y and Cui B: Identification of potential long non-coding RNA biomarkers associated with the progression of colon cancer. Oncotarget 8: 75834-75843, 2017.

16. Zapf A, Brunner E and Konietschke F: A wild bootstrap approach for the selection of biomarkers in early diagnostic trials. BMC Med Res Methodol 15: 43, 2015.

17. Toloşi L and Lengauer T: Classification with correlated features: Unreliability of feature ranking and solutions. Bioinformatics 27 1986-1994, 2011.

18. Wang Q and Liu X: Screening of feature genes in distinguishing different types of breast cancer using support vector machine. Onco Targets Ther 8: 2311-2317, 2015.

19. John B, Enright AJ, Aravin A, Tuschl T, Sander C and Marks DS: Human microRNA targets. PLoS Biol 2: e363, 2004.

20. Huang da W, Sherman BT and Lempicki RA: Systematic and integrative analysis of large gene lists using DAVID bioinformatics resources. Nat Protoc 4: 44-57, 2009.

21. Takebe N, Miele L, Harris PJ, Jeong W, Bando H, Kahn M, Yang SX and Ivy SP: Targeting Notch, Hedgehog, and Wnt pathways in cancer stem cells: Clinical update. Nat Rev Clin Oncol 12: 445-464, 2015

22. Nwabo Kamdje AH, Takam Kamga P, Tagne Simo R, Vecchio L, Seke Etet PF, Muller JM, Bassi G, Lukong E, Kumar Goel R, Mbo Amvene J, et al: Developmental pathways associated with cancer metastasis: Notch, Wnt, and Hedgehog. Cancer Biol Med 14: 109-120, 2017.

23. Culp TD, Budgeon LR, Marinkovich MP, Meneguzzi G and Christensen ND: Keratinocyte-secreted laminin 5 can function as a transient receptor for human papillomaviruses by binding virions and transferring them to adjacent cells. J Virol 80 : 8940-8950, 2006.

24. Yu J, Wang Y, Dong R, Huang X, Ding S and Qiu H: Circulating microRNA-218 was reduced in cervical cancer and correlated with tumor invasion. J Cancer Res Clin Oncol 138: 671-674, 2012.

25. Kogo R, How C, Chaudary N, Bruce J, Shi W, Hill RP, Zahedi P, Yip KW and Liu FF: The microRNA-218 Survivin axis regulates migration, invasion, and lymph node metastasis in cervical cancer. Oncotarget 6: 1090-1100, 2015.
26. Li Y, Liu J, Yuan C, Cui B, Zou X and Qiao Y: High-risk human papillomavirus reduces the expression of microRNA-218 in women with cervical intraepithelial neoplasia. J Int Med Res 38: 1730-1736, 2010.

27. Scheffner M, Huibregtse JM, Vierstra RD and Howley PM: The HPV-16 E6 and E6-AP complex functions as a ubiquitin-protein ligase in the ubiquitination of p53. Cell 75: 495-505, 1993.

28. Ye H, Yu X, Xia J, Tang X, Tang L and Chen F: MiR-486-3p targeting ECM1 represses cell proliferation and metastasis in cervical cancer. Biomed Pharmacother 80: 109-114, 2016.

29. Sun L, Jiang R, Li J, Wang B, Ma C, Lv Y and Mu N: MicroRNA-425-5p is a potential prognostic biomarker for cervical cancer. Ann Clin Biochem 54: 127-133, 2017.

30. Ding H, Wu YL, Wang YX and Zhu FF: Characterization of the microRNA expression profile of cervical squamous cell carcinoma metastases. Asian Pac J Cancer Prev 15: 1675-1679, 2014.

31. Patterson EE, Holloway AK, Weng J, Fojo T and Kebebew E: MicroRNA profiling of adrenocortical tumors reveals miR-483 as a marker of malignancy. Cancer 117: 1630-1639, 2011.

32. Yang ZG, Ma XD, He ZH and Guo YX: miR-483-5p promotes prostate cancer cell proliferation and invasion by targeting RBM5. Int Braz J Urol 43: 1060-1067, 2017.

33. Song Q, Xu Y, Yang C, Chen Z, Jia C, Chen J, Zhang Y, Lai P, Fan X, Zhou X, et al: miR-483-5p promotes invasion and metastasis of lung adenocarcinoma by targeting RhoGDI1 and ALCAM. Cancer Res 74: 3031-3042, 2014.

34. Pan X, Wang R and Wang ZX: The potential role of miR-451 in cancer diagnosis, prognosis, and therapy. Mol Cancer Ther 12: 1153-1162, 2013.

35. Liu D, Liu C, Wang X, Ingvarsson S and Chen H: MicroRNA-451 suppresses tumor cell growth by down-regulating IL6R gene expression. Cancer Epidemiol 38: 85-92, 2014.

36. Kleemann M, Bereuther J, Fischer S, Marquart K, Hänle S, Unger K, Jendrossek V, Riedel CU, Handrick R and Otte K: Investigation on tissue specific effects of pro-apoptotic micro RNAs revealed miR-147b as a potential biomarker in ovarian cancer prognosis. Oncotarget 8: 18773-18791, 2017. International (CC BY-NC-ND 4.0) License. 